\title{
OBSERVATIONAL EVIDENCE FOR POLAR SPOTS
}

\author{
KLAUS G. STRASSMEIER \\ Institut für Astronomie \\ Universität Wien \\ Türkenschanzstraße 17 \\ A-1180 Wien, Austria
}

\begin{abstract}
Are results from ill-posed problems, like Doppler-imaging, conclusive at all ?" and "Could polar spots be simple image-reconstruction artifacts?" are often asked questions. Although I can not definitively answer them, I will present observational evidence for the existence of cool starspots at or very near a stellar rotation pole.
\end{abstract}

\section{Introduction}

The notion of polar spots stems from the first Doppler image of HR 1099 by Vogt \& Penrod (1983) and has been confirmed now for 18 out of 27 late-type stars with Doppler maps (see Appendix Table 1). Despite that these maps were generated by seven different groups of investigators two major points of criticism remain:

- All information on polar spots comes from Doppler imaging which itself is based on the periodic variations in spectral line profiles. The inversion of these line profiles to a two-dimensional temperature image needs information on the "undisturbed" shape and strength of the local line profile: what if the adopted local line profile is incorrect?

- "Why doesn't the Sun show polar spots?" is the obvious second point of criticism - although more a phenomenological one than one against polar starspots, but nevertheless a "nasty" question!

We are just at the beginning of our understanding of the formation of polar spots on rapidly-rotating stars (see Schüssler, this proceedings). We simply do not know enough yet to actually make observable predictions, and I feel 
we should first summarize current observational results before attempting answering the above questions.

\section{A brief survey of Doppler images}

Table 1 lists all published (photospheric) Doppler images of late-type stars (several more are currently in work), their time of observation, the variable type and M-K classification, rotation parameters, the amplitude of the lightcurve in case this information is used for the Doppler map, the inclination $i$ of the stellar rotation axis and whether a polar spot (P), or high-latitude $(\mathrm{H})$ or low-latitude $(\mathrm{L})$ spots were seen. The 27 stars in Table 1 consist of 12 RSCVn binaries, 4 T Tauri objects, 3 FK Comae stars, 2 W UMa contact systems, 4 young single dwarf stars, and 2 BY Draconis binary. Their rotation periods range between 19.4 days for the $\mathrm{K} 1$ giant $\sigma$ Gem to the 0.31 days of the G2V W-UMa system YY Eri. Respectively, the unprojected equatorial rotational velocities range from $165 \mathrm{~km} \mathrm{~s}^{-1}$ for FK Comae to $26 \mathrm{~km} \mathrm{~s}^{-1}$ for II Peg. Furthermore, almost all evolutionary stages are present: from classical and weak-lined T Tauri stars to evolved MK-class III giants, singles and binaries, the latter with and without mass transfer. Consequently, a wide range of stellar radii has been observed: between the $0.77 \mathrm{R}_{\odot}$ for the dM1e stars in YY Gem to the $16 \mathrm{R}_{\odot}$ of YY Men. Finally, the effective surface temperatures of the stars in Table 1 span from an estimated cool $4000 \mathrm{~K}$ for DF Tau and YY Gem to a warm $6000 \mathrm{~K}$ for the W-UMa system AE Phe.

\section{Evidence from line-profile modeling}

\subsection{THE QUEST FOR CORRECT TRANSITION PROBABILITIES}

Figure 1 presents several line-profile fits to the observed solar Ca I 6439.075$\AA$ line taken from Kurucz's et al. (1984) solar atlas. The theoretical profiles were computed with the solar model of Kurucz (1991) $(5770 \mathrm{~K}, \log g=4.55$, $\mathrm{He} / \mathrm{H}=0.1, \xi=1.0 \mathrm{~km} \mathrm{~s}^{-1}$ ), and his most recent line list, incorporating altogether 40 million lines. The line-synthesis program of Stift (1995) is being used to compute chunks of spectra from a solution of the equation of transfer from a grid of 72 depth points including micro- and macroturbulence as well as rotation.

The only parameters left for fitting the solar profiles were the transition probabilities $(\log g f)$ of the mapping lines and their blends. Our best estimates are (in parenthesis: our $\log g f$ value | Kurucz-CD value): Ca I $6439.075(+0.47 \mid+0.47)$, Fe I $6430.852(-1.85 \mid-1.85)$, Fe I $6411.647(-0.32 \mid-$ $0.82)$, Fe I $6393.602(-1.50 \mid-1.62)$, Li I $6707.850(+0.18 \mid+0.18)$. Obviously, the $\log g f$ 's used for imaging are not so unrealistic as sometimes said - and 


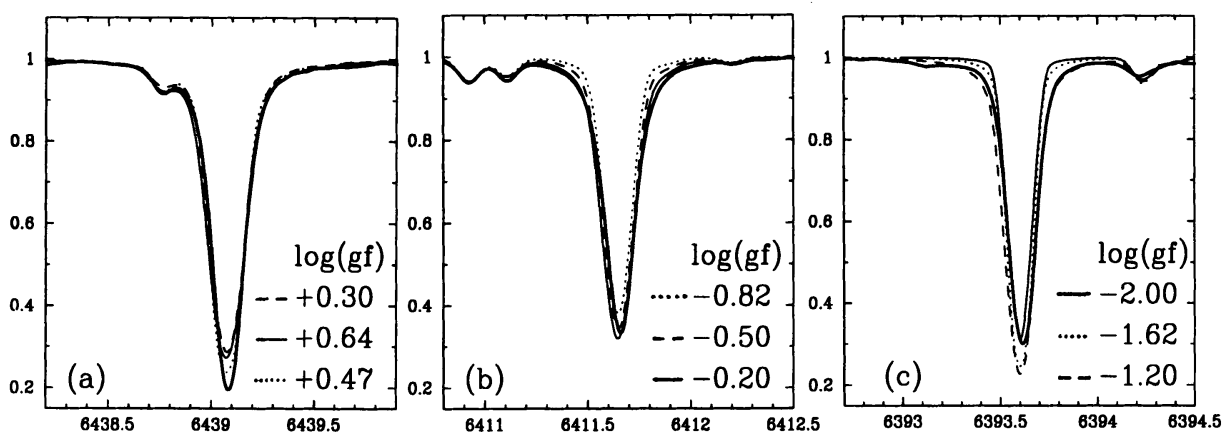

Figure 1. Examples of our comparison of theoretical line profiles with the observed solar spectrum. Left: Ca I 6439.075. Middle: Fe I 6411.647. Right: Fe I 6393.602.

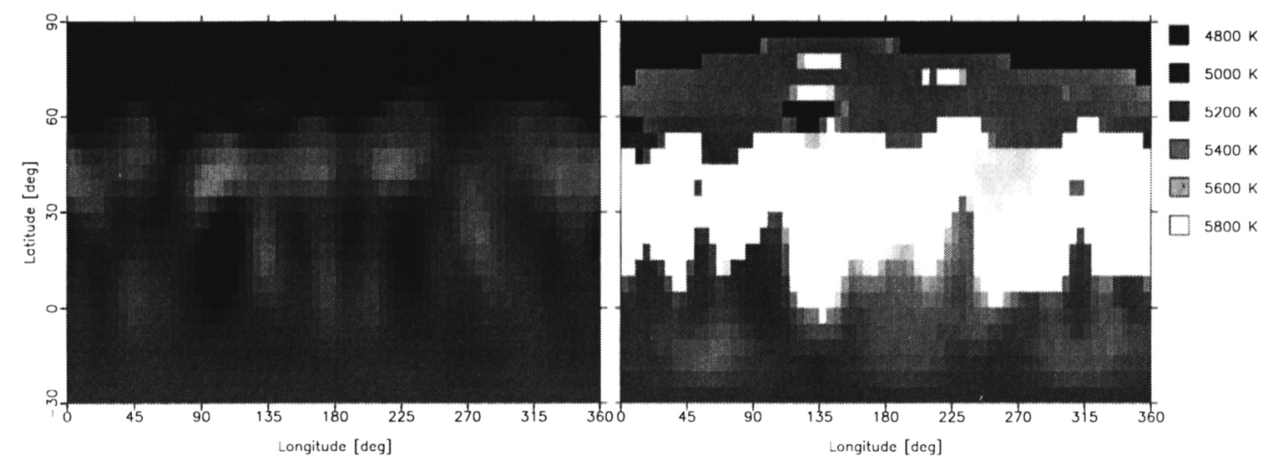

Figure 2. Two Ca I Doppler maps of HD 199178 reconstructed with incorrect transition probabilities: $\log g f=+0.30$ (left) and +0.64 (right) instead of +0.47 .

this fact strengthens the reliability of Doppler maps and consequently the existence of polar spots.

No depth dependence of microturbulence could be taken into account in our computations, which might explain part of the misfit of the core of the strong $\mathrm{Ca}$ I line and of the wings in the weaker lines. The C-type asymmetry of both solar lines in Fig. 1 (thick line) is the typical result of surface convection in granular cells. Note that the theoretical Ca lines are too shallow for the observed solar line depth and would - if severely enhanced in active stars - produce the opposite effect than a polar spot!

Figure 2 presents two Doppler maps of HD 199178 reconstructed from "real" data with all their intrinsic faults, like incomplete phase coverage or possible errors in the continuum setting, but computed with wrong transition probabilities $(\log g f=+0.64$ and +0.30 instead of +0.47$)$. A weaker $\log g f$ has - at least in the case of Ca I 6439 - only little influence on the 
generated map (the $\chi^{2}$ of the line-profile fit is 0.039 , almost identical to the fit with +0.47 ) corresponding to the map in Fig. 2 (left), while a stronger $\log g f$ has a disastrous effect on the reconstructed map (the $\chi^{2}$ of the fit is 0.109 ) because the local profiles become too broad and the code is forced to put a hot band around the star (Fig. 2 right). Amazingly though, and this is the point of the whole exercise, a polar spot is still recovered!

\subsection{UNKNOWN ELEMENTAL ABUNDANCES}

Could an incorrectly adopted abundance result in a spurious polar spot? And how different could the abundance be to be neglected in the lineprofile inversion? Figure 3 compares two Doppler maps for HD 199178 from Ca I 6439 , one with "correct" abundance $(-5.15)$ and one with an artificial overabundance of 0.2 dex. Both maps were computed from altogether eight blends with equivalent widths between 3 and $15 \mathrm{~m} \AA$. Once again, the polar spot is clearly recovered and needed by the data. Note that an artificial underabundance would actually strengthen a polar cap-like feature.

\subsection{BLENDED LINE PROFILES}

Unruh \& Cameron (1995) already showed that the neglect of blends in the wings of the mapping line could lead to spurious banding at high latitudes, and eventually to a polar-spot look-alike if the equivalent width of the blend is large enough. However, these were model calculations. What about real observations? Figure 4 shows two Ca I-6439 maps of HD 199178. Both were reconstructed without blends. One with "correct" abundance and the other with an overabundance of again 0.2 dex. The missing equivalent width due to the blends is thereby compensated for with the artificial overabundance. A polar spot is the result in any case.

\subsection{DEPENDENCE OF LINE STRENGTH ON TEMPERATURE}

The most recent images listed in Table 1 were already generated by taking into account that the local line profile from a spot is different than from the surrounding warmer photosphere. Again, polar spots were reconstructed e.g. for HR 1099! For further details on the temperature dependence of spectral lines see the articles of Gray (this proceedings) and Stift \& Strassmeier (1995).

\subsection{DO WE USE THE CORRECT MICROTURBULENCE?}

Figure 5 compares three synthetic Ca I 6439 line profiles from a $T_{\text {eff }}=5500$ and $\log g=2.5$ model atmosphere and microturbulences of $\xi=0,1$, and 2 


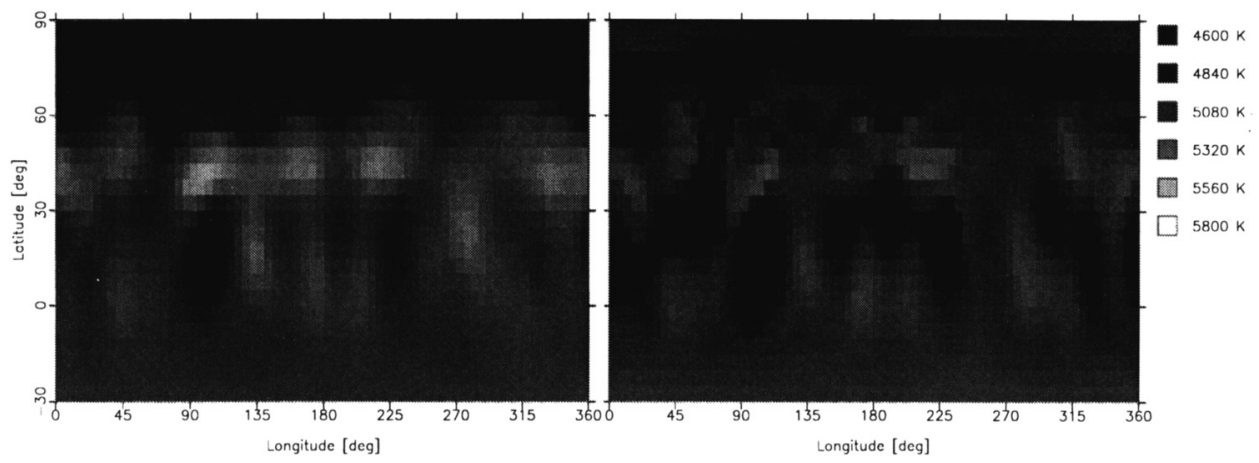

Figure 3. Doppler images of HD 199178 with "correct" input abundance (left image) and an artificial overabundance of 0.2 dex (right image). Both images were computed from Ca I 6439- $\AA$ including eight blends.

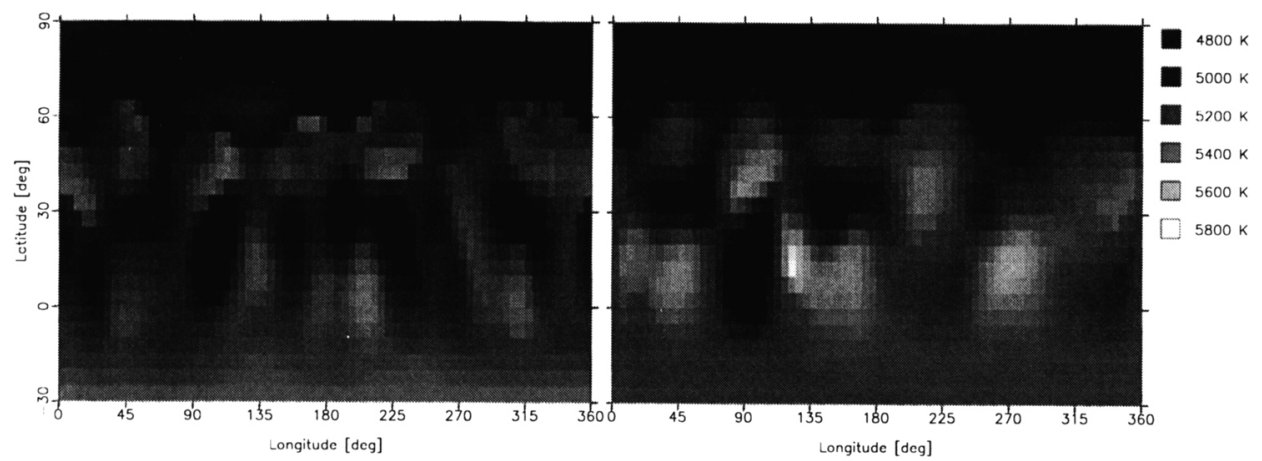

Figure 4. Doppler images of HD 199178 reconstruced from the same data set as in Fig. 2 but without blends. Left: with correct abundances. Right: with an artificial overabundance of 0.2 dex.

$\mathrm{km} \mathrm{s}^{-1}$. In Fig. 5 notice the different profile shape for the very weak line, an Fe I line to the blue of the $\mathrm{Ca}$ I line, and the strong Ca I 6439 line! So we may suspect that a wrongly adopted microturbulence has a profound effect on our reconstructed Doppler image. But does it especially affect the stellar polar regions? Figure 6 is a reconstruction of the same set of observations of HD 199178 as in Figs. 2, 3 and 4 but without microturbulence at all. One map is with correct $\log g f(+0.47)$ and the other with an abnormally large $\log g f(+0.64)$. The latter was obtained by optimizing the quality of the profile fit but in no case, however, got the $\chi^{2}$ closer to the "correct" line-profile fit (with $\xi=2 \mathrm{~km} \mathrm{~s}^{-1}$ ) than roughly a factor two. Due to the smaller equivalent width of the local line profile the inversion code comes up with a generally cooler surface to compensate for the missing equivalent 

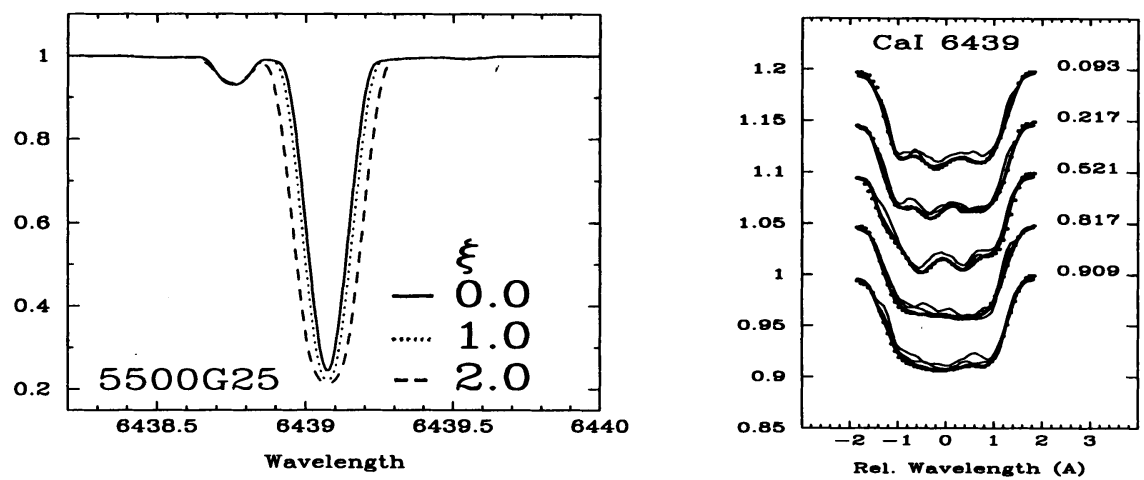

Figure 5. Left: The effect of microturbulence $\xi$ on the CaI 6439 line. Right: HD 199178 fits with zero microturbulence and correct $\log g f$ (thin line: worst fit), a too large a log $g f$ of +0.64 (thin line: slightly better fit) and the best fit with $\xi=2 \mathrm{~km} \mathrm{~s}^{-1}$ and $\log g f=+0.47$ (thick line). The dots are the observations.
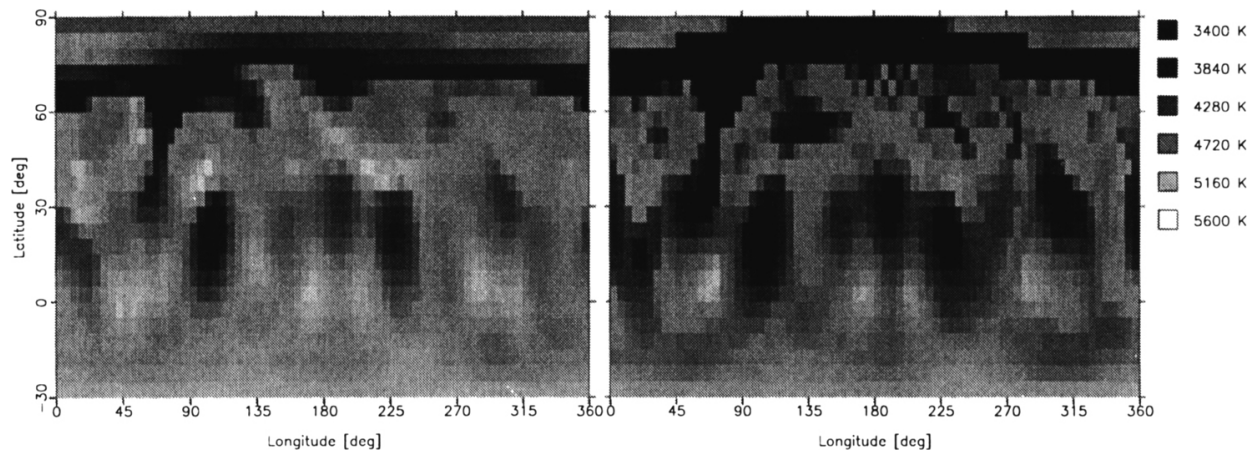

Figure 6. Doppler images of HD 199178 reconstructed with zero microturbulence and correct $\log g f$ (left) and too strong a $\log g f$ (right), respectively.

width (note the different temperature scales in Fig. 6).

Actually, a cool polar feature is reconstructed for HD 199178 fairly independent of the adopted microturbulence. The cap-like structure, however, changes to something like a circumpolar ring when a grossly incorrect $\log g f$ and $\xi$ are used.

\subsection{INTRINSIC SPOT VARIABILITY}

All previously discussed line-profile complications could only show up as a symmetric effect in a Doppler image - for example a circular polar cap or an equatorial ring. Wouldn't it be nice evidence for the reality of polar spots if we had mapped a star at different times with the same mapping code and 
found a polar spot at a particular time and none at some other time? Well, this is exactly the case for the K2 dwarf star LQ Hya (see Table 1) where Saar et al. (1994) reconstructed an asymmetric polar spot in March 1993 but not in May and February 1991 (neither did our own map from February 1991). Obviously, the line profile models couldn't be the cause of the polar feature in 1993. Furthermore, long term variations in the asymmetry of the persistent polar spot of HR 1099 (see Vogt \& Hatzes, this proceedings) are additional evidence of its reality. How could a time-independent symmetric fault produce a time-dependent asymmetric artifact?

\subsection{DEPENDENCE OF THE PROFILE SHAPE ON INCLINATION}

Hatzes et al. (1995) presented a poster at this meeting that dealt with a possible dependence of the shape of observed average line profiles with the inclination of the stellar rotation axis: the more inclined, the flatter the observed line profile. If proven correct, it is further evidence for the existence of polar spots. Our Table 1 tells us that all stars with $i \leq 45^{\circ}$ were reconstructed with a polar spot (except the single attempt by Gondoin (1986) for HR 1099) and thus strengthens Hatzes's conjecture.

Other (secondary) evidence has been presented - mainly from long-term photometry - by, e.g., Oláh et al. (1992)(1991) for HK Lac and V833 Tau, by Pettersen et al. (1992) for BY Dra and EV Lac, by Dempsey et al. (1992) for HD 199178 and HR 1099, by Strassmeier (1990) for EI Eri.

Acknowledgements: It's a pleasure to thank John Rice for numerous discussions on the mapping topic, especially during our long Hawaiian nights, Kolya Piskunov for providing the numerical data of Kurucz's NSO solar atlas, and Steve Vogt for refereeing this paper.

\section{References}

Collier Cameron A., 1995, MNRAS 275, 534

Collier Cameron A., Unruh Y. C., 1994, MNRAS 269, 814

Dempsey R. C., Bopp B. W., Strassmeier K. G., Granados A. F., Henry G. W., Hall D. S., 1992, ApJ 392, 187

Donati J. F., Brown S. F., Semel M. et al., 1992, A\&A 265, 682

Gondoin P., 1986, A\&A 160, 73

Hatzes A. P., 1993, ApJ 410, 777

Hatzes A. P., 1995a, AJ 109, 350

Hatzes A. P., 1995b, in Strassmeier K. G. (ed), Poster Proceedings: Stellar Surface Structure, IAU Symp. 176, Univ. Vienna, p. 87

Hatzes A. P., 1995c, in Strassmeier K. G. (ed), Poster Proceedings: Stellar Surface Structure, IAU Symp. 176, Univ. Vienna, p. 90

Hatzes A. P., 1995d, ApJ 451, 784

Hatzes A. P., Vogt S. S., Ramseyer T. F., Misch A., 1995, in Strassmeier K. G. (ed), Poster Proceedings: Stellar Surface Structure, IAU Symp. 176, Univ. Vienna, p. 9

Hatzes A. P., Vogt S. S., 1992, MNRAS 258, 387 
Hempelmann A., Hatzes A. P., Kürster M., Patkós L., 1995, in Strassmeier K. G. (ed), Poster Proceedings: Stellar Surface Structure, IAU Symp. 176, Univ. Vienna, p. 194

Hubl B., Strassmeier K. G., 1995, in Strassmeier K. G. (ed), Poster Proceedings: Stellar Surface Structure, IAU Symp. 176, Univ. Vienna, p. 96

Jankov S., Donati J.-F., 1995, in Huang L. et al. (eds), Proc. of the 4th Workshop on MUSICOS, ESA-ESTEC, p. 143

Joncour I., Bertout C., Bouvier J., 1994a, A\&A 291, L19

Joncour I., Bertout C., Menard F., 1994b, A\&A 285, L25

Kurucz R. L., 1991, in Davis Philip A. G., et al. (eds), Precision Photometry: Astrophysics of the Galaxy, Schenectady, Davis, p. 27

Kurucz R. L., Furenlid I., Brault J., Testerman L., 1984, Solar Flux Atlas from 296 to $1300 \mathrm{~nm}$, NSO Atlas No. 1

Kürster M., Dennerl K., 1993, in Linsky J. F. \& Serio S. (eds), Physics of Solar and Stellar Coronae, Kluwer Academic Publishers, Netherlands, p. 443

Kürster M., Hatzes A. P., Pallavicini R., Randich S., 1992, in Giampapa M. S. \& Bookbinder J. A. (eds.), Seventh Cambridge Workshop, Cool Stars, Stellar Systems, and the Sun, PASPC 26, p. 249

Kürster M., Schmitt J. H. M. M., Cutispoto G., 1994, A\&A 289, 899

Maceroni C., Vilhu O., van't Veer, F., Van Hamme W., 1994, A\&A 288, 529

Noah P. V., Bopp B. W., Fekel F., 1987, in Linsky J. L. \& Stencel R. E. (eds), Fifth Cambridge Workshop, Cool Stars, Stellar Systems, and the Sun, Springer, p.506

Oláh K., Budding E., Butler C. J., Houdebine E. R. et al., 1992, MNRAS 259, 302

Oláh K., Pettersen B. R., 1991, A\&A 242, 443

Petrov P. P., Gullbring E., Gahm G., et al., 1995, in Strassmeier K. G. (ed), Poster Proceedings: Stellar Surface Structure, IAU Symp. 176, Univ. Vienna, p. 217

Pettersen B. R., Olah K., Sandmann W. H., 1992, A\&AS 96, 497

Piskunov N. E., Huenemoerder D. P., Saar S. H., 1994, in Caillault J. P. (ed), Eight Cambridge Workshop, Cool Stars, Stellar Sytems, and the Sun, PASPC 64, p. 658

Piskunov N. E., Tuominen I., Vilhu O., 1990, A\&A 230, 363

Ramseyer T. F., Hatzes A. P., Jablonski F., 1995, AJ 110, 1364

Rice J. B., Strassmeier K.G., 1996, A\&A, (in press)

Saar S. H., Piskunov N. E., Tuominen I., 1994, in Caillault J. P. (ed), Eight Cambridge Workshop, Cool Stars, Stellar Systems, and the Sun, PASPC 64, p. 661

Stift M. J., 1995, private comm.

Stift M. J., Strassmeier K. G., 1995, in Strassmeier K. G. (ed), Poster Proceedings: Stellar Surface Structure, IAU Symp. 176, Univ. Vienna, p. 29

Stout N., Vogt S. S., 1996, this proceedings

Strassmeier K. G., 1990, ApJ 348, 682

Strassmeier K. G., 1994, A\&A 281, 395

Strassmeier K. G., 1996, A\&A, (in press)

Strassmeier K. G., Rice J. B., Wehlau W. H. et al., 1991, A\&A 247, 130

Strassmeier K. G., Rice J. B., Wehlau W. H. et al., 1993, A\&A 268, 671

Strassmeier K. G., Welty A. D., Rice J. B., 1994, A\&A 285, L17

Strassmeier K. G., Dempsey R. C., Rice J. B., 1996, A\&A (submitted)

Unruh Y. C., Collier-Cameron A., Guenther E., 1995, in Strassmeier K. G. (ed), Poster Proceedings: Stellar Surface Structure, IAU Symp. 176, Univ. Vienna, p. 93

Unruh Y. C., Collier Cameron A., 1995, MNRAS 273, 116

Vogt S. S., 1988, in Carel de Strobel G. \& Spite M. (eds.), IAU Symp. 132, The Impact of Very High $S / N$ Spectroscopy on Stellar Physics, Kluwer Academic Publishers, p.253

Vogt S. S., Hatzes A. P., 1990, in Tuominen I., Moss D., Rüdiger G. (eds), IAU Coll. 130, The Sun and Cool Stars: Activity, Magnetism, Dynamos, Springer Verlag, p. 297

Vogt S. S., Hatzes A. P., 1996, this proceedings

Vogt S. S., Penrod G. D., 1983, PASP 95, 565 
TABLE 1. A list of Doppler images of late-type stars

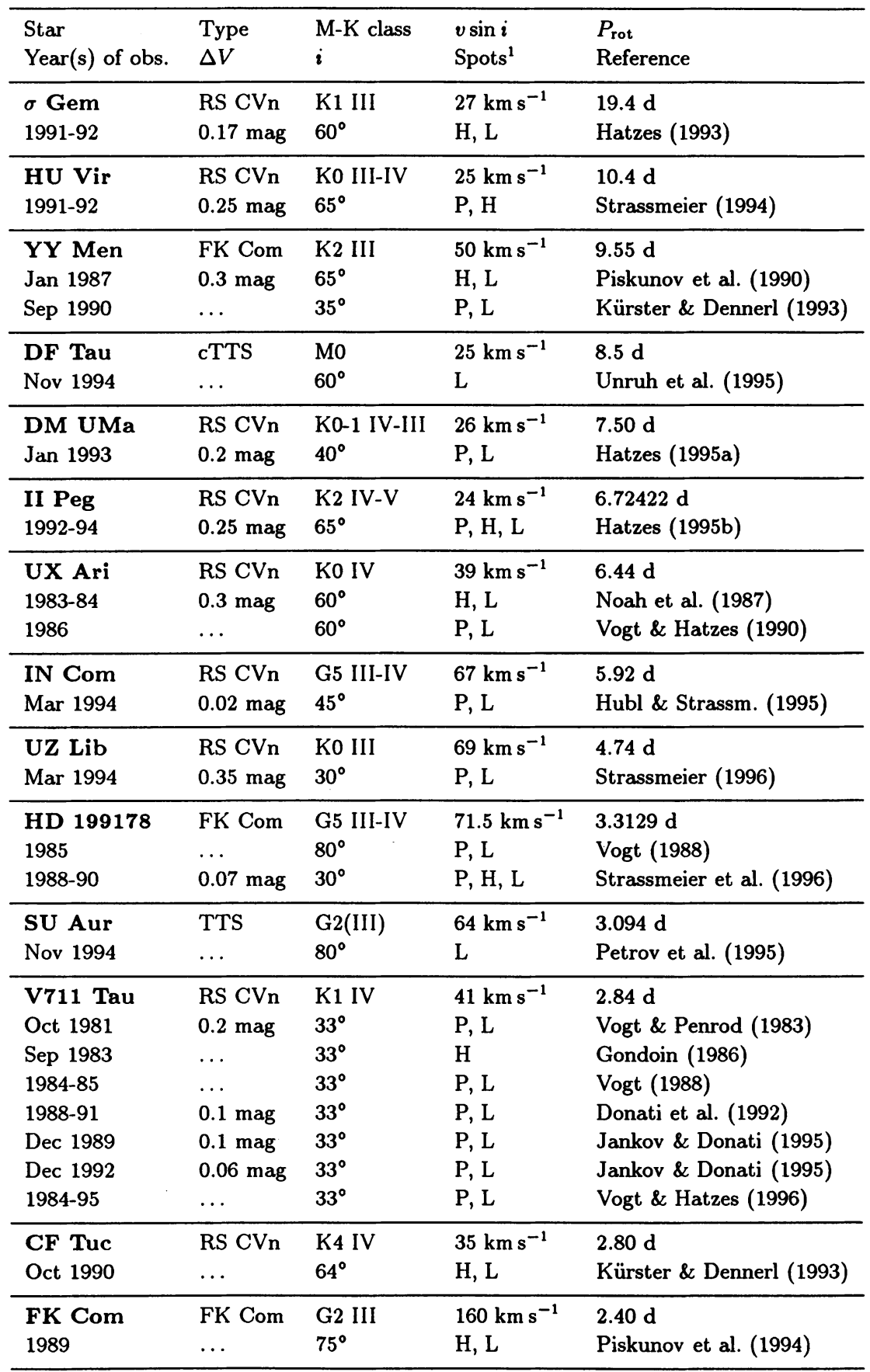


TABLE 1. (continued)

\begin{tabular}{|c|c|c|c|c|}
\hline $\begin{array}{l}\text { Star } \\
\text { Year(s) of obs. }\end{array}$ & $\begin{array}{l}\text { Type } \\
\Delta V\end{array}$ & $\begin{array}{l}\text { M-K class } \\
i\end{array}$ & $\begin{array}{l}v \sin i \\
\operatorname{Spots}^{1}\end{array}$ & $\begin{array}{l}P_{\text {rot }} \\
\text { Reference }\end{array}$ \\
\hline EI Eri & RS CVn & G5 IV & $50 \mathrm{~km} \mathrm{~s}^{-1}$ & $1.95 \mathrm{~d}$ \\
\hline $1984-87$ & $0.1 \mathrm{mag}$ & $46^{\circ}$ & $\mathrm{P}, \mathrm{L}$ & Hatzes \& Vogt (1992) \\
\hline $1987-88$ & $0.15 \mathrm{mag}$ & $46^{\circ}$ & $\mathrm{P}, \mathrm{H}, \mathrm{L}$ & Strassmeier et al. (1991) \\
\hline V410 Tau & WTT & K4 & $77 \mathrm{~km} \mathrm{~s}^{-1}$ & $1.872 \mathrm{~d}$ \\
\hline Jan 1990 & $0.4 \mathrm{mag}$ & $70^{\circ}$ & $\mathrm{P}$ & Joncour et al. (1994b) \\
\hline Nov 1992 & $0.37 \mathrm{mag}$ & $70^{\circ}$ & $\mathrm{H}, \mathrm{L}$ & Strassmeier et al. (1994) \\
\hline Dec 1993 & $0.5 \mathrm{mag}$ & $70^{\circ}$ & (P), H, L & Rice \& Strassmeier (1996) \\
\hline 1993-94 & $\ldots$ & $54^{\circ}$ & (P), H, L & Hatzes (1995d) \\
\hline HD 155555 & RS CVn & KO V-IV & $29 \mathrm{~km} \mathrm{~s}^{-1}$ & $1.68 \mathrm{~d}$ \\
\hline Sep 1990 & $\ldots$ & $55^{\circ}$ & $\mathrm{P}, \mathrm{L}$ & Kürster et al. (1992) \\
\hline LQ Hya & single & $\mathrm{K} 2 \mathrm{~V}$ & $27 \mathrm{~km} \mathrm{~s}^{-1}$ & $1.61 \mathrm{~d}$ \\
\hline Feb 1991 & $0.1 \mathrm{mag}$ & $70^{\circ}$ & $\mathrm{H}, \mathrm{L}$ & Strassmeier et al. (1993) \\
\hline Feb 1991 & $\ldots$ & $75^{\circ}$ & $\mathrm{H}, \mathrm{L}$ & Saar et al. (1994) \\
\hline May 1991 & $\ldots$ & $75^{\circ}$ & $\mathrm{H}, \mathrm{L}$ & Saar et al. (1994) \\
\hline Mar 1993 & $\ldots$ & $75^{\circ}$ & $\mathrm{P}, \mathrm{L}$ & Saar et al. (1994) \\
\hline HD 283572 & WTT & G8 IV-V & $80 \mathrm{~km} \mathrm{~s}^{-1}$ & $1.55 \mathrm{~d}$ \\
\hline Feb 1993 & $0.1 \mathrm{mag}$ & $60^{\circ}$ & $\mathrm{P}$ & Joncour et al. (1994a) \\
\hline YY Gem AB & BY Dra & dM1e & 48 & $0.8142822 \mathrm{~d}$ \\
\hline Feb 1993 & $\ldots$ & $87^{\circ}$ & $\mathrm{H}, \mathrm{L}$ & Hatzes (1995c) \\
\hline SV Cam & RS CVn & G2V-IV & $117 \mathrm{~km} \mathrm{~s}^{-1}$ & $0.59 \mathrm{~d}$ \\
\hline $1993-94$ & $0.07 \mathrm{mag}$ & $90^{\circ}$ & $\mathrm{H}, \mathrm{L}$ & Hempelmann et al. (1995) \\
\hline V471 Tau & BY Dra & $\mathrm{K} 2 \mathrm{~V}$ & $91 \mathrm{~km} \mathrm{~s}^{-1}$ & $0.5211 \mathrm{~d}$ \\
\hline 1992 & $\ldots$ & $90^{\circ}$ & $\mathrm{P}, \mathrm{H}$ & Ramseyer et al. (1995) \\
\hline Dec 1993 & $0.1 \mathrm{mag}$ & $90^{\circ}$ & $\mathrm{P}, \mathrm{H}$ & Ramseyer et al. (1995) \\
\hline AB Dor & single & $\mathrm{KO} \mathrm{V}$ & $91 \mathrm{~km} \mathrm{~s}^{-1}$ & $0.514790 \mathrm{~d}$ \\
\hline Feb 1989 & $0.1 \mathrm{mag}$ & $60^{\circ}$ & $\mathrm{H}, \mathrm{L}$ & Kürster et al. (1994) \\
\hline Jan 1992 & $\ldots$ & $60^{\circ}$ & $\mathrm{P}, \mathrm{H}, \mathrm{L}$ & Collier-C. \& Unruh (1994) \\
\hline Dec 1992 & $\ldots$ & $60^{\circ}$ & $\mathrm{P}, \mathrm{H}, \mathrm{L}$ & Collier-Cameron (1995) \\
\hline HII 3163 & Pleiades & MOV & $70 \mathrm{~km} \mathrm{~s}^{-1}$ & $0.42 \mathrm{~d}$ \\
\hline Dec 1993 & $\ldots$ & $30^{\circ}$ & $(\mathrm{P}), \mathrm{H}, \mathrm{L}$ & Stout \& Vogt (1996) \\
\hline HII 686 & Pleiades & $\mathrm{K} 5 \mathrm{~V}$ & $64 \mathrm{~km} \mathrm{~s}^{-1}$ & $0.397 \mathrm{~d}$ \\
\hline Dec 1993 & $\ldots$ & $42^{\circ}$ & (P), H, L & Stout \& Vogt (1996) \\
\hline AE Phe & W UMa & $<$ G0 $>$ & $?$ & $0.3623718 \mathrm{~d}$ \\
\hline Nov 1989 & $\ldots$ & $88^{\circ}$ & $\mathrm{H}, \mathrm{L}$ & Maceroni et al. (1994) \\
\hline Nov 1990 & yes & $87^{\circ}$ & $\mathrm{H}, \mathrm{L}$ & Maceroni et al. (1994) \\
\hline YY Eri & W UMa & $\langle\mathrm{G} 2 \mathrm{~V}\rangle$ & $?$ & $0.31249510 \mathrm{~d}$ \\
\hline Nov 1989 & $\ldots$ & $82^{\circ}$ & $\mathrm{H}, \mathrm{L}$ & Maceroni et al. (1994) \\
\hline Nov 1990 & yes & $82^{\circ}$ & $\mathrm{H}, \mathrm{L}$ & Maceroni et al. (1994) \\
\hline
\end{tabular}

${ }^{1} \mathrm{P}=$ Polar spot, $\mathrm{H}=$ High-latitude spots, $\mathrm{L}=$ Low-latitude spots 